Gerrenlojes Gut betradtet unb von Senowertern, arbeitern mit Stumpi und Stiel auggerottet. 2 ngefäet ober aufgeforitet wird bie Fläche nicht,

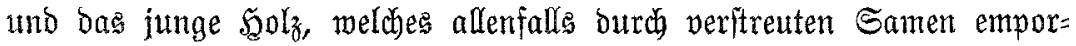
Eeimen will, wirb vom Bieh zeritört. Dieje Fläche ift weder 21cterland noch Meibe nocb Mald, fie liegt im troftlofen 3 uftanbe ba und bringt feinen Muten. Rännte einer berartigen Bermüftung nidyt auf irgend eine Weife vorgebeugt werben? Der Bildbeitano unjerer Bauernwälber ift faum nennenswert. Febe und Şiriche find barin nicht vorhanben, und

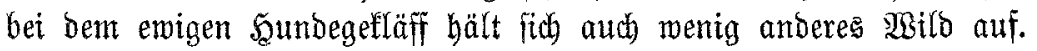

Ditpreußen ift an $\mathfrak{B a ̈ l b e r n ~ n i c h t ~ a r m ; ~ a l l e i n ~ b i e ~ S c a b j u c h t ~ d e r ~}$ Menidien mut alles gewinnbringende rajther und rübfichtslojer aus wie frïher, und ba $\mathfrak{S}_{0} \mathfrak{l}_{z}$ ein jehr begehrter uno pretsmerter 2 trtffel ift, io greift man bie $\mathfrak{B a ̈ l d}$ er vielfach rüffichtslos an, augebeno von bem Grundiate: Mach uns bie Sintflut!

Unjere $\mathfrak{A}$ tworbern binterließ̉en ung umfangreiche, gutbeftandene

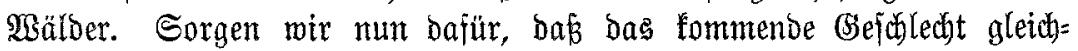
falle gute Waloungen von uns erbalte. Der Nuben ber $\mathfrak{B a ̈ l b e r}$ ift jo mannigfaltig uno großj, ba $\mathfrak{p}$ es fid mohl ber Mühe lohnt, fie zu fidonen und zu pflegen. Das ßeijpiel ift Der beite Rebrmeifter. Man follte alio nidht auf jene Balbbefitber jeben, die für ben Fortbejtand nichts thun. Der umfitbtige, fparjame uno vormärts ftrebenbe Ranowirt findet vielfach

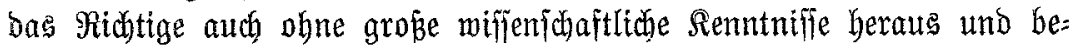
tritt willig bie Bahnen berjenigen, benen ber Befte eines guten $\mathfrak{W a l b e s}$ zum Stolze gereicht.

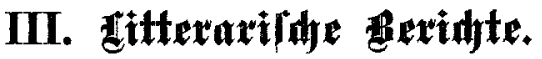

গr. 7.

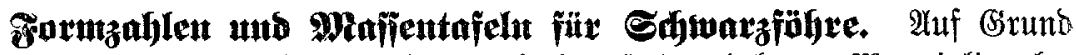
ber von ber 2 . foritlithen Berjuchsanitalt erbobenen Materialten be= arbeitet won Songenieur $\mathfrak{R} a r l$

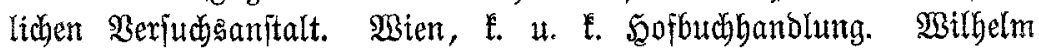
Frivef. 1893.

Die vorliegende nur 32 Drudfeiten umfaffende Schrift ift nur ein

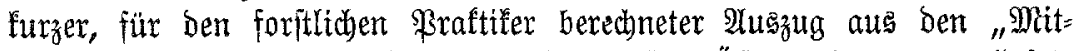
teilungen aus bem forftlichen Serjuchswejen Sitterretiss, XV. Sgeft". Sesteres Seft bringt natmlich eine eingebende Bearbeitung ber Form: zablen unb Mafjentafeln für bie Edjwarzföhre auf 110 Quartjeiten, mit 
6 photographifben Iafeln und zwei albbilduntgen im Iexte und umfá̧t aljo bas ganze Sruntomaterial, weldese zut Serarbeitung biente, für ben praftijchen Bebraudb ber Formzahlen und Maffentafeln aber bireft nidbt notmendig ift.

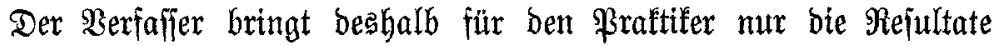
ber Unterfuchung in Frorm von "auggeglichenen" Formzablen uno fertigen Mafjentafeln in bequemerem Dltavformat und jojidft benjelben einen mur

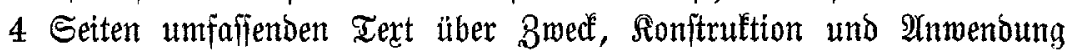
ber $\mathfrak{x} a f e l n$ voraus. Da das Material zu ben Iafeln bem Schinarz= föhrengebiete in Sitederöfterreich entnommen ift, io miro bie fleine Schrift ntatürlid aud nur für foldhe Forftwirte von praftifidem Werte fein, welche in biejem $\mathfrak{B a l}$ ogebiete mirtidaften.

Dáa bie Mafientafeln in erfter Sinie den $3^{\text {medten }}$ ber Beftandes=

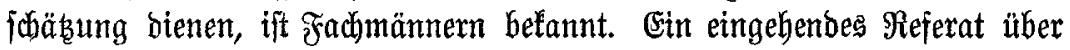
Das grȫere $\mathfrak{B e r f}$ aus welchem das vorliegende Schriftchen entîtanden ift, finbet fich Seite 487 u. f. von 1894 biejer 3eitiörift. F. $\mathfrak{B a u r}$.

\section{Mr. 8.}

Pritteilungen aus ber Etantsforftwewwaltutg Bayems. Sceraus: gegeben vom Egl. Staateninifterium Der Finanzen, Minifterialforit= abteilung. 1. 5̧eft. München 1894.

Mit vorlieaendem, erfiten Seefte werden bie jeit 1846 unter bem Titel "foritwirtichattliche Mittetlungen" uno fpäter unter ber \$ezeidymung "Forftliche Mitteilungen" allen baverifden und beutichen Forftwirten wohl befannten, zmanglofen Mitteilungen ber bayerijben Staateforit= verwaltung, bie bis zum Jabre 1876 erjojienen, wieder aufgenommen.

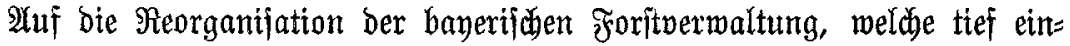

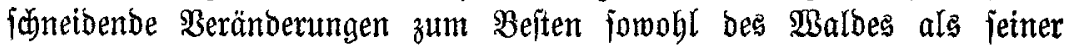

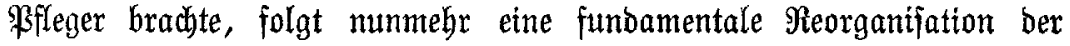
bisher vormiegend übliden Methoden ber wirtfłhaftlidben Bebanblung uno

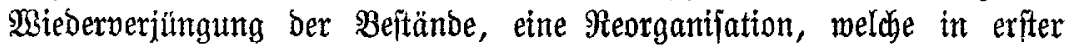
Sinie ben natürlichen Berbältriffen von Rlima, \$oben uno Şolzant gleid)

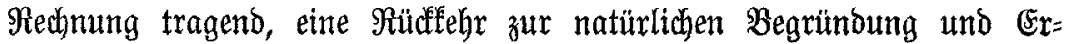
ztehung bes $\mathfrak{B a l b e s}$, zum Mifchwalde in Berjüngungsformen bedentet, auf beren Wert feit Şahren unjer Mltmeifter Geheimrat Ģayer in ber Sitteratur bingemiejen hat. Daß eine forgiame Forjtwermaltung über

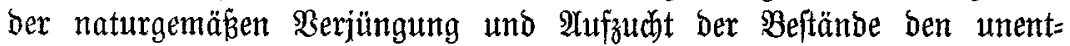

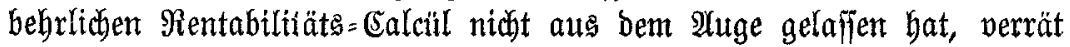
jebe Beile ber neuen \$sirtidjaftsregeln. 
Bündig und llar find biefe Regein entworfen uno fo jowterig bie= jelben exjdeinen mögen, je tiefer ntan einoringt um fo einfactjer, um fo

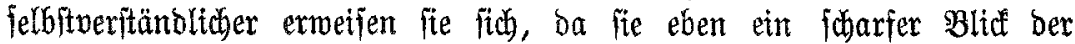
Natur, ben Bäumen felbft abgelejen hat.

Dex I. Ieil enthält gleidjam bie Quaberiteine, auf benen fï̀) bie

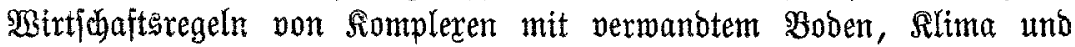
Şolzarten alffbauten laffen; bie $\mathfrak{B i r t j}$ aftsregeln für bas ehentalige $\mathrm{fgl}$.

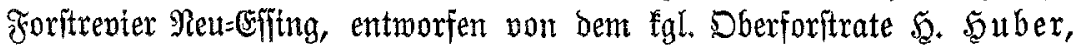
enthalten Ferlen ber Beobad)tung auf ben verfobiedenen (Sebieten ber

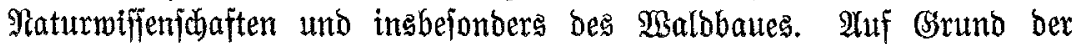

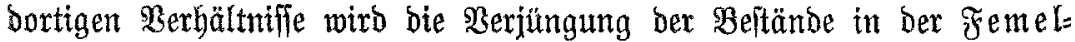

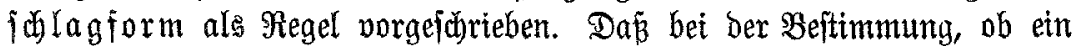
Эormuds braudfbar ober nidft jei, bie ftrengiten $\mathfrak{A n f o r d e r u n g e n ~ a n ~ D i d h t e , ~}$

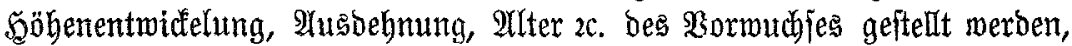
verbient bent vollen \$eifall; beffer lein $\mathfrak{B o r m u d b}$ als ein niubt allen $\mathfrak{2} \mathfrak{n}=$ forberungen gemügender. Mur jelten werben Berbältnifje uns zmingen, auth mit bem ioblecteren Bormutife vorlieb zu nehmen.

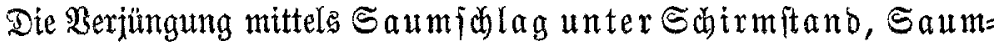
fohirmidläge ober hier Saumfemelidgläge genant mit einer gra= phifchen Darftellung Des Berfabrens in jehr ítark geneigtem Terrain. Dieje Methobe hat in jüngfter Beit noch eine weitere Ergänzung erfabren barin,

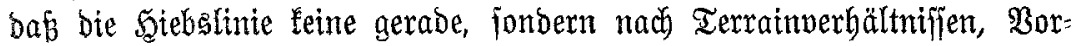
mudbspartieen aud eine ausgebuchtete sinie baritellen barf.

Der II. Ieil bejajt fíd mit ben Grundlagen für eine umfaffende Maldftandsrevijion im Silzer Irift= regeln bezitehen fï̀ auf bie 4 Şauptbeitandşormen bes Kompleres, näm= (ic) 1. auf ben aus Budben, Tanmen unb Fituten ober Fichten und sitchen

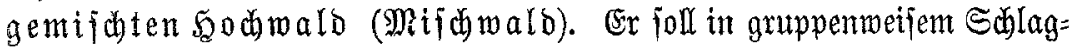
betriebe, Femelidblagmintfchaft, bebandelt werben; bie Serjüngung wirb beendet burch einen Saumbieb, weldyer bie Beitandoreite zwijojen ben frei geittellten Gruppen binwegnimmt. $2 \mathfrak{H}$ fiteilen Scängen wiro eine Gruppenverjüngung zonenweije, bei übermiegen pon fiobten ber regel= mäbige Saumjiblag eingelegt.

2. F̧ü bie reinen oder mit Iannen gemifăten Fichtenbejtände wiro bas gleiche $\mathfrak{B i n t i j h a f t s p e r f a b r e n ~ a b o p t i e r t . ~}$

3. Für $\mathfrak{A} u$ walbungen (Fithtenmaldungen) ift évenfalls, mo bie Ratur fofon bas Material bietet, eine natürliche Berjüngung in Femeijislag ober Saumidalag beabiiditigt.

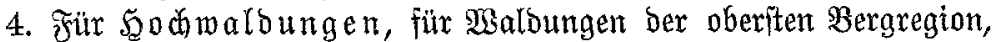

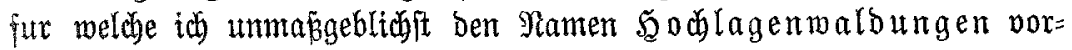




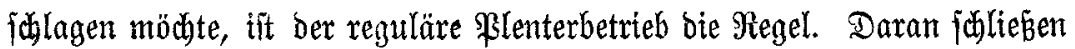
jith bann Borichriften für bie jadjliche uno formelle Behandhung ber Maloftandsrevifionsarbeiten.

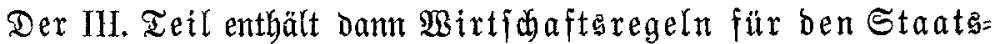

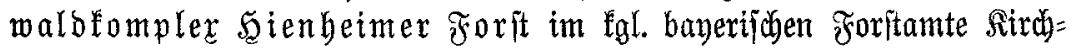
heim Süb.

Dort find 3 Seauptbeitanogformen ausgej fieden, nänlidb:

1. Der reine Eidjenbeftand mit $\mathfrak{B} u$ chenunterbau, fünttlid angelegt, bie älteitten erịt 50 jährig.

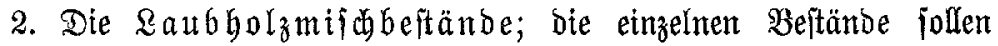

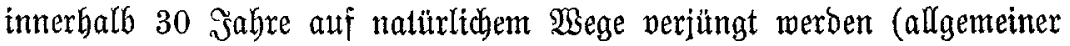
Berjüngunggzeitraum). Der Fpeztelle $\mathfrak{B}$ erjüngungszeitraum, die Beit, welche vom eriten ânhiebe einer Gruppe bis zur Şinmegnahme ber leksten Schirmrefte über ber Grruppe verläuft, wechjelt je nach Şolzarten, Boben=

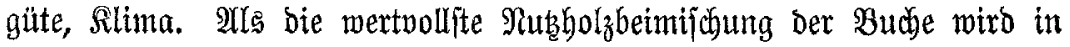

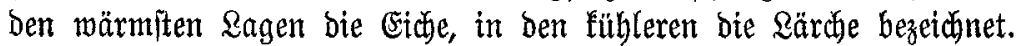

3. Die Rabelbolzbeftände find je nach S̈rtlidfeten teils in Femelijhlag, teils in Saumichlag, teils in bem fombinierten Berfahren

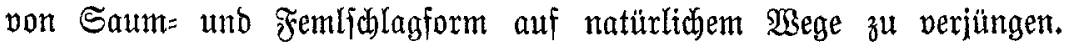

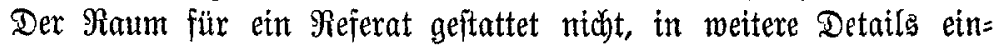
zugeben; es ift bies bier audid gar nicht nötig; jedem bayerifchen Frorit= manne find bie neuen Mitteilungen unentbebrlich, für bie außerbayerijeden Foritleute bringen fie eine Fitlle von neuen Thatiachen und nachabmungs= werten $\mathfrak{B i n f e n . ~ M o ̈ g e ~ j e d e r ~ f i e ~ p r u ̈ f e n ~ u n d ~ f u ̈ r ~ f e i n e ~ \Re e r h a ̈ l t n i f f e ~ b a s ~}$ Pafjendite baraus behalten. Dann aber möge man nidbt vergeffen, mit uns ber bayerifchen Staatsforftwerwaltung bantbar zu fein, für bie libe

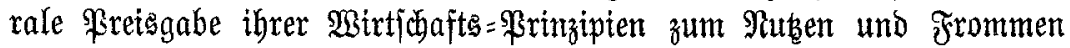
aller.

Mand.

$\mathfrak{i r} .9$.

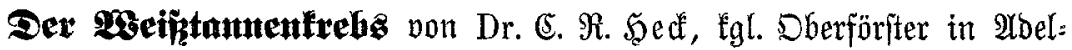

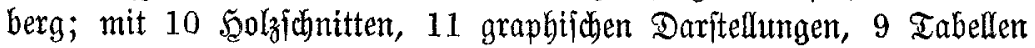
und 10 sidutorudétafeln. Berlin 1894. §. Springer.

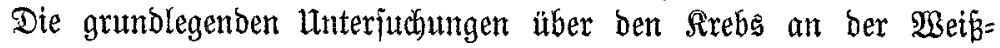
tanne, über ben 3ujammenbang won Şerenbejen uno Stammbeulen, über

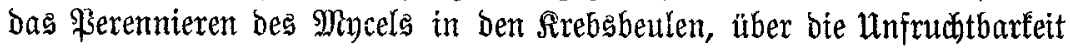
Des Mivels in ben $\mathfrak{B}$ eulen und andere Eigentumltubeeiten rübren von be $\mathfrak{B a r y}$ her, ber jeine $\mathfrak{A}$ rbeit in ber wentg zugänglidben, botanijchen 3eitung bereits 1867 veröffentlichte. Seit biejer Beit bis zum Sabre 1892 ift in bie Biologie biejes Pilzes leine wejentliche Alufbellung georungen. 
Das meifte finto nur gelegentlich in Bezug auf J̈nfeftion ausgepronchente Bermutungen; vor allem ift es nidht gelungen, mit ben Sporen bes Serenbejents wieberum Sexenbejen zut erzeugen ober ben 3 ulammentang bes Serenbejents mit einer Ieleutofiporenform auf einer anderen $23 i r t s=$ pflanze aufzufinder.

2Uth über bie $\mathfrak{A}$ rt ber Snfeftion, ob von ber austreibenden snofpe ausgehent, wie $\mathfrak{B} e \mathrm{ije}$ in jeiner trefflithen 2 bhandlung annimmt, oder

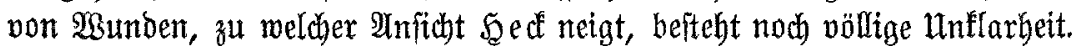
lltber alle bieje widhtigen Eigentümlichéten bes şilzes bringt auth ber

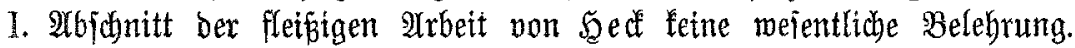

Biel leichter ift natürlich bie Unterjuctung ber anatomifonen $\mathfrak{B} e$

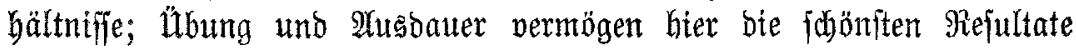
zu erzielen, während für bie Erforichung ber biologifichen Berbältniffe vor allem neben naturwiffenfchaftlicher Schulung binreichende Beit unb eine

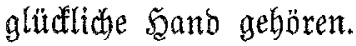

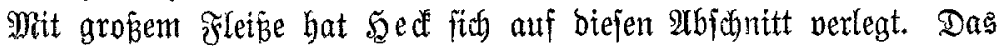
Beripringen ber bicéen Borfenrinde an ben Srebsbeulen ber Tannen mit bem geiteigerten Buwadbs ber Srebsbeulen in 3ujammenthang zut bringen, ericheint nicht zuläifig. Selbit an ben riefigen Fropfmafern, wie fie be= jonbers bei ßappeln burth ein ganz enormes Dicfenwadbstum entiteben,

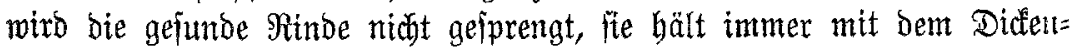

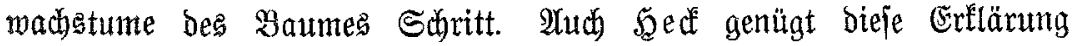
augenfdeinlid nidht, benn er nimmt für bieje Berreiß̧ungen, bie für ben

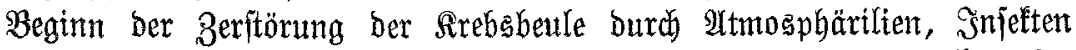

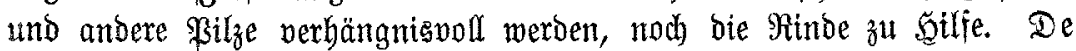

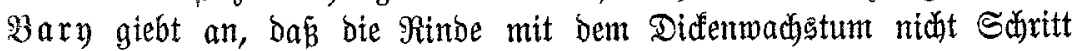

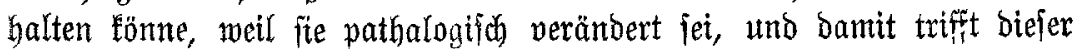

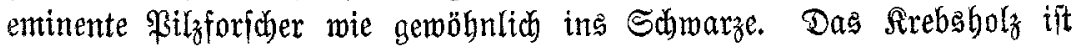
jebr viel jobmerer als bas gefunde Tannenbolz; aber wie bie beigebradten

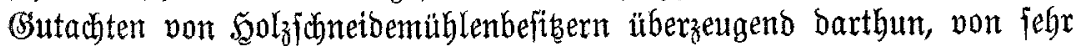

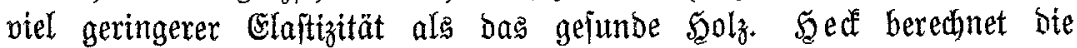
Sdiäbigung bes Stammes burch bent Bentenpilz bis zu 20 p(Et. bes Mertes.

Die demifuen Unterjudungen hat Frof. Dr. Seubert in Tü= bingen vorgenommen. Umfaffende Eryebungen jodann hat $\mathfrak{s e d}$ über

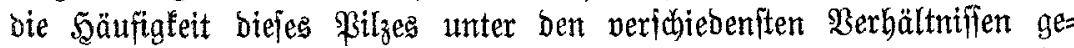

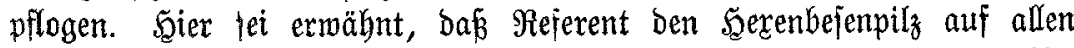
japantifyen Tannen fontatieren fonnte. Intereffant ijt ein Iannengebiet in Deutidiland, wo ber Srebs bis jeşt vollitändig fehlt, nämlich in Dit= friesland, mo es baubare Beftânbe giebt; bie Tanne ift bort mur auf 


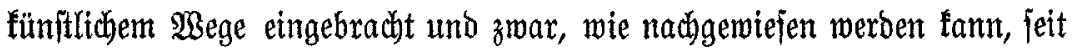

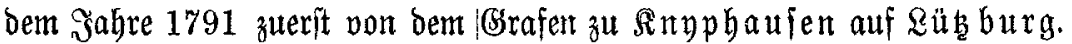

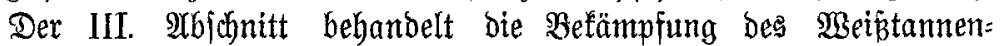
frebjes, meldhe, folange bie Biologie bes Urbebers der ßrantheit noch fo unvollfommen befannt ift, eine unvolitändige bleiben muk; benn alle

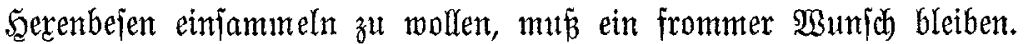

Man fann bem Serfaffer nur beiftimmen, wenn er bas h్geraus= nebmen ber pon bem Silge im Stamme befallenen Sntoiviouen bet ben

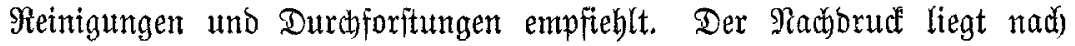

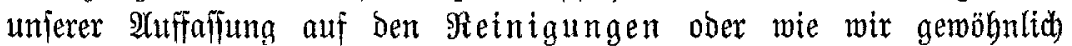
fagen auf ber Sdlagpflege, bei biejer fömen aus ben bichten, frei geftellten Scoritent alle Srebspflanzen herantgebauten meroen, ba bie anderen

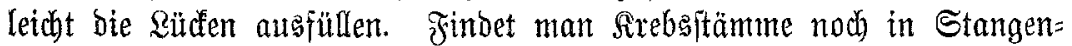
bölzern, fo find fie ein Bemets von verfäumter Shlagflege. Die Durbs: foritungen fönnen nidbt den 3 wed haben, den $\mathfrak{B i}_{\mathfrak{z}}$ zu befämpjen, denn bie Beulen an ben Stämmen find unfrudthtbar; bie Durdforitung beab= fitutigt mut einen Stamm zu retten, ehe er burd) bie $\mathfrak{B}$ eulen zu viel an Bert eingebüß̄̌ hat.

Die nad \$hotographieen hergeitelten Beilagen mit vielen 2 bbildsungen

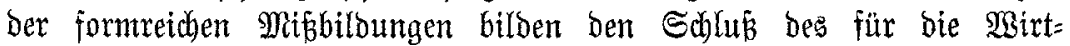
juafter in Tamnenrevieren lehrreichen Buthes, das überoies eine gewandte

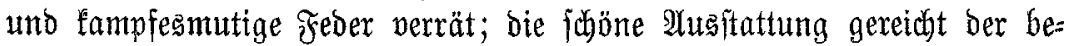
wäbrten Serlagefirma zur Eabre.

$\mathfrak{M a y n}$.

\section{शr. 10.}

Nomenclator coleopterologicus. Eine etnmologifiche Graflärıng fämt=

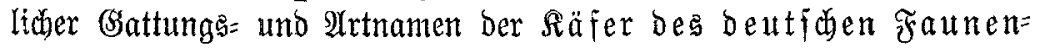
gebietes, von Stegmuno Shenkling. Frankfurt a/M. Berlag poit $\mathfrak{6}$. Becthyolo 1894. 224 Seiten $\mathfrak{B r e i s} 4 \mathscr{M}$.

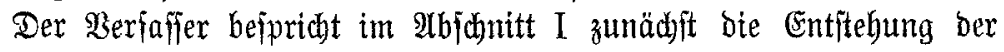
binären ober binomen Momenclatur, wie fie burdh Sinné eingefühnt und

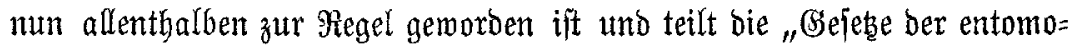
logifchen Romenclatur" mit, weldbe auf ben entomologijoben Rongrép zu Dresden ant 23. Maat 1858 feftgeiebt wurben.

Snt Ilbichnitt II "Grattungen und lintergattungen" fübrt er in alpha= betijber Dronung alle Gattungsnamen ber zur beutfoen Fauna geb̆örigen Räfer an und giebt bie Erffärung für deren Arbjtammung, während

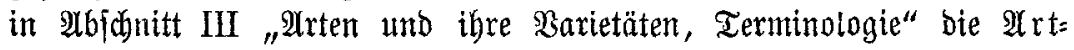


namen in gleicher Beije behandelt werben. ${ }^{1}$ ) Urbjonitt IV zählt bie

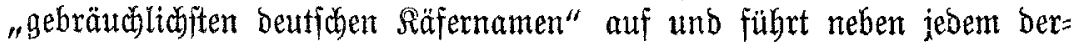

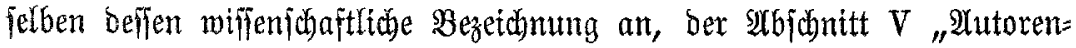
verzeichnig" enolich weît bie Mamen ber in ber entomologijichen Pomen=

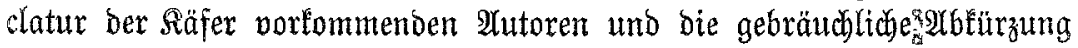
derielben nach.

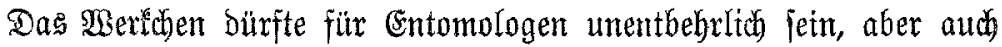

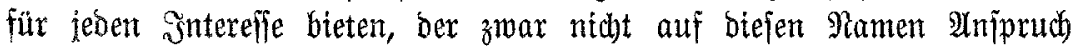
erbeben fann, fich abex aus siebhaberei ober $\mathfrak{B}$ eruf - wie bie Forft= leute - mehr ober weniger mit ber Räferwelt bejhäftigt. Es bietet aud bem im Griectifichen und \&ateinifhen minder Bemanderten bie Mög=

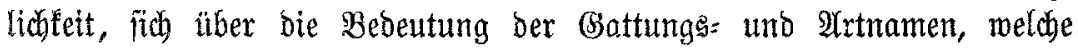
ja in ben meiften Föallen für äupere sericheinung ber Rebensweife des betr. Snjettes bezeichnend find, fomie über bie 2 tusprache berjelben, melche burch bie befannten Beichen tenntlich gemadht ift, zu belehren.

$\mathfrak{M r} .11$.

Winfe fïu Stufünger. Ein Reitfaden für angebende Büchter und Riebhaber von Raffehunden. Zujammengeftellt uno herausgegeben von

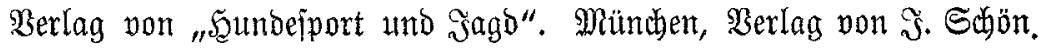
1894. 88 Seiten.

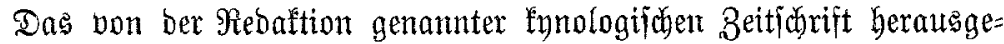

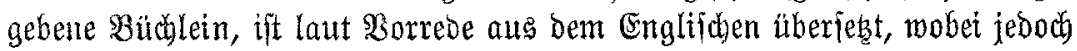
bie Eriahrungen beuticher Büdter teilmeife an Etelle Des Driginals treten.

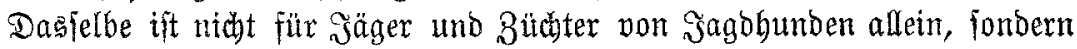

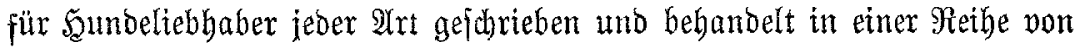

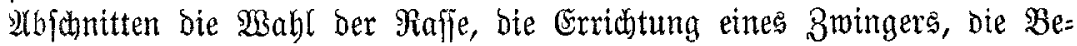
Gandlung von Bubththünbinnen, bie häuftger auftretenden Şunbefranflyeiten,

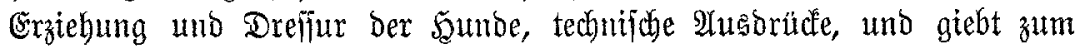
Sullus die 2rbbilbumgen ber in Deutichland hauptiäblich verbreiteten

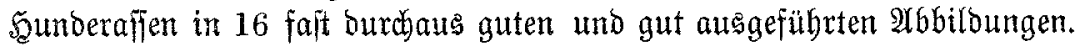

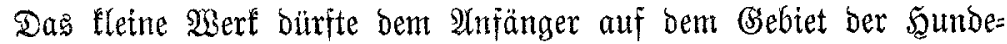
3ucht uno Şunbebaltung bieten, was ber Titel peripricht. Winfe über Erziehung und Behandang von Şunden jeber $2 \mathfrak{n t}$, und manthes bier

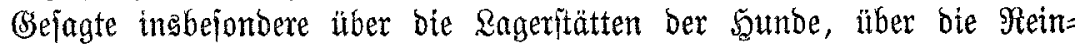

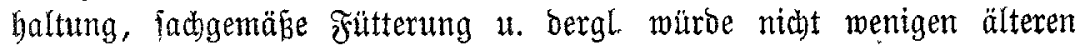

1) Es möge bier bemerft jein, baj̄ in 2 ent Fuñoten ebenfall gegeben find. 
Saunbebejtbern zur Beadtung zu empfehlen jein - es wirb in biejer

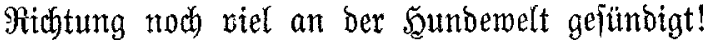

Eine etroas Iogijchere Bebandlung bes Stoffes wäre ba uno bort zu empieblen gemejen: fo iit z. 2 . bie Staupe eimmal in einem bejonderen

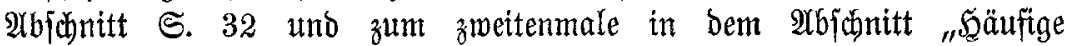
Srantheiten" auf S. 40 behandelt; ebenjo die $\Re a ̈ u b e$, einmal S. 37 unter bem nichtsjagenden Titel "Berjobiebenes" und nochmals $\subseteq$ S. 41 unter ben Fुındefranflyeiten. Der Gruno für bieje boppelte Befprectung

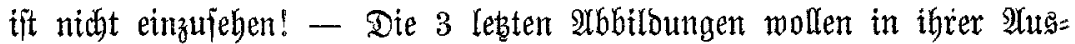
führung nur wenig zu ben vorbergebenden viel hübfoberen paffen.

Šundefreunden bürfte das Biudblein wohl zul empfehlen jein.

$\mathfrak{F}$.

\section{Altotizen.}

Die forftlehranjtalt Eifenach.

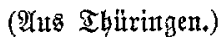

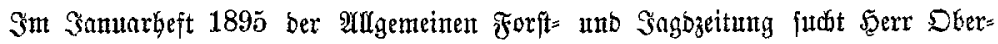

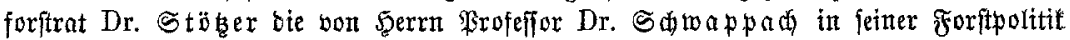

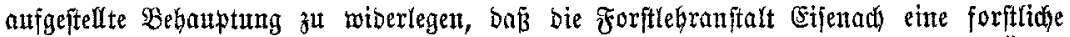

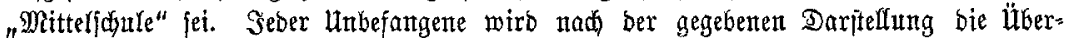

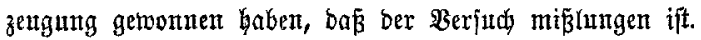

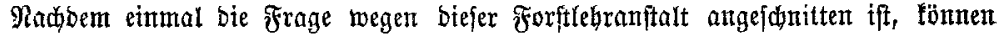
wir uns nidjt verfagen, aud einige sBemertungen barüber z̆t madjent. Dabei wolle aber

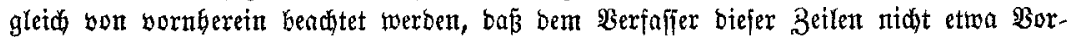

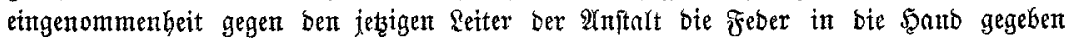

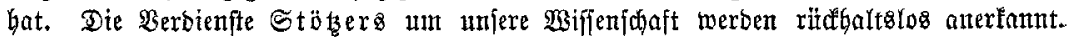

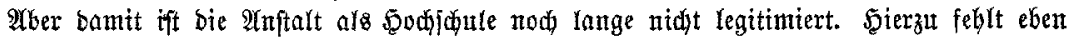

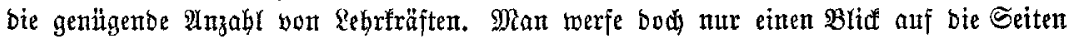
19 und 424 bes Forrifalenders, II. Tetrs, umb man wirb finben, baj ber Unterridit

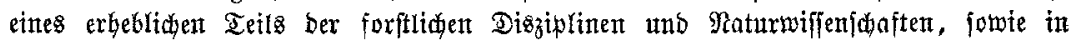

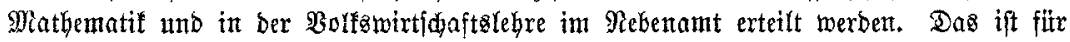

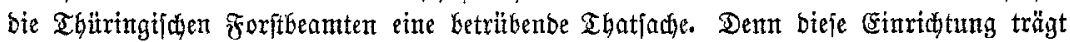

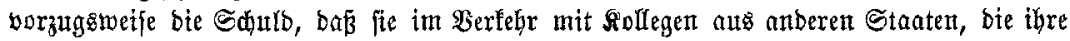

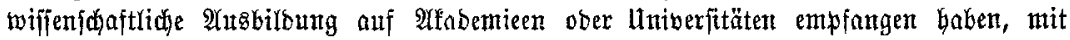

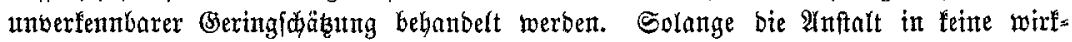

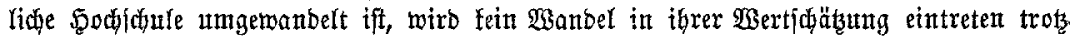

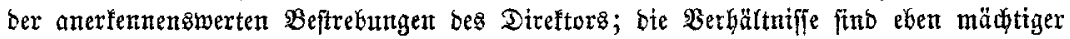
als ber gutte Biffe.

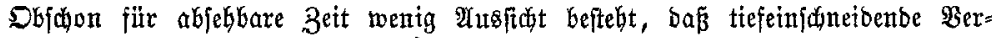
änberungen an ber $\mathfrak{A}$ niftaft borgenommen werben, wollent twir bod eimmal ben finan=

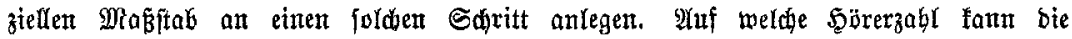
Sodule au\& ben Staatêforfiberwoltungen redinen? Sie pflegt befudit zu merben bon 